\title{
A Comparison of Two Reference Point Methods in Multiple Objective Mathematical Programming
}

\author{
John Buchanan \\ Department of Management Systems \\ University of Waikato \\ Hamilton, New Zealand \\ jtb@waikato.ac.nz \\ Lorraine Gardiner \\ Department of Management \\ Auburn University \\ Auburn, AL 36839, USA \\ gardilr@mail.auburn.edu
}

\begin{abstract}
When making decisions with multiple criteria, a decision maker often thinks in terms of an aspiration point or levels of achievement for the criteria. In multiple objective mathematical programming, solution methods based on aspiration points can generate nondominated solutions using a variety of scalarizing functions. These reference point solution methods commonly use a scalarizing function that reaches down from the ideal solution, in a direction specified by the aspiration point. Conversely, a similar scalarizing function can push out from the nadir point toward a specified aspiration point. In this paper we explore the structure of these two reference point approaches, examine the discrepancy between resulting solutions and consider the effect of problem framing on decision maker behaviour.
\end{abstract}

\section{Introduction}

Reference point methodology provides the foundation for many interactive search procedures in multiple objective programming. The concept of a reference point is consistent with Simon's [6] description of satisficing decision making where a decision maker tends to have targets or goals in mind while proceeding toward a decision. This satisficing approach is specifically accommodated in the reference point methods of Wierzbicki [9] and Korhonen and Wallenius [4]. However, reference point formulations have also been used in "non-satisficing" models such as [7], where a reference point formulation is used as a mechanism for implicitly optimizing a decision maker's value function. Two forms of reference points exist: aspiration points (desirable levels of achievement) and reservation points (levels of achievement that should be attained, if at all possible). In this study, we focus our attention on aspiration points. 
Wierzbicki [9] produced seminal research on reference point methods, including an investigation of the characteristics of various achievement functions for allowing the search for attractive nondominated solutions to be controlled by reference points. These achievement functions were designed to have a significant advantage over goal programming by producing only nondominated, or Pareto-optimal, solutions. An important class of achievement functions considered in this paper have formulations similar to the weighted Tchebycheff distance metric.

In addition to their desirable structural features, reference point methods have also appeared useful from a methodological or operational perspective. They provide the "shell" for a simple, decision maker controlled solution method that does not require the decision maker to conform to any particular requirements or axioms. The Pareto Race method of Korhonen and Wallenius [4], where a decision maker simply moves over the efficient search in a relatively unstructured search for a most preferred solution, is one such example. Buchanan [2] has proposed an even simpler solution method, also using a Tchebycheff achievement function.

In this paper we compare two formulations, both using Tchebycheff-based achievement functions and aspiration points, but having quite different philosophies. One seeks to "push out" from an undesirable solution while the other "reaches down" from a desirable but unachievable solution. It is known that these two formulations produce different solutions, given identical input (that is, the same aspiration point) from a decision maker. We shall investigate the patterns of discrepancy between solutions from these two formulations and show that the resulting solutions can be significantly different, for the same aspiration point.

From a behavioural perspective, the choice of reference point can considerably affect a decision maker's perception of the resulting solution. Proverbs 27:7 states the point succinctly, "He who is full loathes honey, but to the hungry even what is bitter tastes sweet." If, for example, the reference point is the nadir or worst solution, then any resulting solution is a significant improvement. Conversely, if the reference point is the ideal or best solution, any resulting solution must be worse. Even if these two solutions are the same, comparison with their respective reference points is likely to result in different "values" being placed on them. The two opposing reference points for the formulations (pushing out from an undesirable solution or reaching down from a desirable solution) is similar to the concept of framing, which has been discussed in the behavioural decision making literature. Image Theory, a descriptive decision making model proposed by Beach and Mitchell [1], provides a useful illustration of framing. They suggest that a decision maker has images or schemata which represent, in some way, his view or values. With the Image Theory model, the first stage of any decision is a Compatibility Test where each alternative in turn is assessed to determined its "fit" with the decision maker's images. Usually these images will be, in some sense, ideal. Clearly, the choice of image significantly affects the choice of alternative. More specifically, Tversky and Kahneman [8] show that how a problem is framed (in their example, whether the same problem is stated negatively or positively) significantly affects decision maker choice. With respect to reference points, the pushingout and reaching-down formulations can be viewed as different ways of framing the decision problem, for the same aspiration point. To examine the behavioural effects of this framing, we also present some results of a study on how human decision makers respond to solutions from these two formulations, given the same aspiration point. 


\section{Terminology and Definitions}

The multiple objective mathematical programming problem can be stated as:

$$
\begin{aligned}
\operatorname{MAX} & F(\boldsymbol{x})=\left[f_{1}(\boldsymbol{x}), f_{2}(\boldsymbol{x}), \ldots, f_{q}(\boldsymbol{x})\right] \\
\text { s.t. } & \boldsymbol{x} \in X
\end{aligned}
$$

where $\boldsymbol{x}$ is an n-dimensional vector of decision variables; $F$ is a vector-valued function comprising $q$ distinct, concave objective functions of $\boldsymbol{x} ; X \subset R^{n}$ is the feasible set of constrained decisions; and $Z=F(\boldsymbol{x}) \in R^{q}$ is the image of $X$ in objective function or criterion space. We further assume that set $X$ comprises $m$ constraints of the form, $X=\left\{x \in R^{n}\right.$ : $\left.g_{j}(\boldsymbol{x}) \leq 0, j=1,2, \ldots, m\right\}$. The operator MAX indicates that each objective function is to be maximized over $X$.

The set of nondominated solutions $N$ consists of those criterion vectors $z \in Z$ which are not dominated by another criterion vector in $Z$. A criterion $z^{1}$ is said to dominate solution $z^{2}$ if

$$
\begin{aligned}
& z_{k}^{2} \leq z_{k}^{1} \text { for all } k=1,2, \ldots, q \\
& z_{j}^{2}<z_{j}^{1} \text { for at least one } j \in\{1,2, \ldots, q\}
\end{aligned}
$$

Two assumptions are made about the preferences of the decision maker. First, preferences are assumed to monotonic; that is, given the MAX operator for each objective, "more is always better than less." Second, a decision maker is presumed to want to consider only non-dominated solutions. Let $U_{k}$ and $M_{k}$ be the maximum and minimum values of each objective $k$ over the non-dominated set $N$.

The vector of $U_{k}$ values $\boldsymbol{U}$ is usually referred to as the ideal point while the vector of $M_{k}$ values $\boldsymbol{M}$ is often labelled the nadir point. The $U_{k}$ and $M_{k}$ values can be used to rescale the criterion vectors in order to provide commensurable measurement of all objectives over $N$. The function $h_{k}(\boldsymbol{x})$ maps any criterion vector $z \in N$ onto the range [0,1]. Using this transformation, $\boldsymbol{h}(\boldsymbol{M})=(0,0, \ldots, 0)$, and $\boldsymbol{h}(\boldsymbol{U})=(1,1, \ldots, 1)$ and $h_{k}(\boldsymbol{x})=\left(f_{k}(\boldsymbol{x})-M_{k}\right) /\left(U_{k}-M_{k}\right)$. The criterion space is therefore scaled and $N$ is contained within a $q$-dimensional unit hypercube anchored at the origin of the nonnegative orthant. The remaining discussion assumes this transformation.

\subsection{PU and PM Formulations}

Programs PU and PM are defined as follows. PU uses a Tchebycheff-based achievement function to minimize the maximum weighted deviation from the ideal solution $\boldsymbol{U}$. Program $\mathrm{PM}$, on the other hand, seeks to maximize the minimum weighted achievement from the nadir solution $\boldsymbol{M}$. For a given set of weights $\boldsymbol{w}$, the PU and PM formulations are:

$$
\begin{aligned}
& \mathrm{PU}(\mathbf{w}): \quad \text { Min } y \\
& \text { s.t. } y \geq w_{k}\left(1-h_{k}(\boldsymbol{x})\right), w_{k} \geq 0, k=1,2, \ldots, q \\
& \boldsymbol{x} \in X \\
& \operatorname{PM}(\mathbf{w}): \quad \operatorname{Max} y \\
& \text { s.t. } y \geq w_{k} h_{k}(\boldsymbol{x}), w_{k} \geq 0, k=1,2, \ldots, q \\
& \boldsymbol{x} \in X
\end{aligned}
$$


The PM formulation has $\boldsymbol{M}$ as its reference point while for PU the reference point is $\boldsymbol{U}$. We distinguish between a reference point such as either $\boldsymbol{M}$ or $\boldsymbol{U}$, which is an integral part of the problem formulation, and an aspiration point, which is an input specified by the decision maker.

Figure 1 shows, in two dimensions, the relationships among these different terms. The set of nondominated solutions is the piecewise linear set ABCD. $\boldsymbol{a}^{1}$ and $\boldsymbol{a}^{2}$ are aspiration points. The line segment $\boldsymbol{A D}$ is the set of points where $h_{1}+h_{2}=1 . \boldsymbol{h}^{* M 1}$ is the PM solution for $\boldsymbol{a}^{l}$ while $\boldsymbol{h}^{* U 1}$ is the PU solution for $\boldsymbol{a}^{1}$; similarly for $\boldsymbol{a}^{2}$. From Figure 1, it can be seen that the discrepancy between PM and PU solutions is a function of the shape of the nondominated set, the location of the aspiration point relative to nondominated set, and the distance of the aspiration point from the line segment $\boldsymbol{L}=\boldsymbol{M U}$. Even in the twodimensional case, it is obvious that the discrepancy between $\boldsymbol{h}^{* M}$ and $\boldsymbol{h}^{* \mathrm{U}}$ can be substantial. It is also worth noting that for aspiration points "underneath" $N$ and not on $\boldsymbol{L}, \boldsymbol{h}^{* \mathrm{U}}$ is closer to the center of $N$ than $\boldsymbol{h}^{* M}$. The reverse is true for aspiration points "above" $N$.

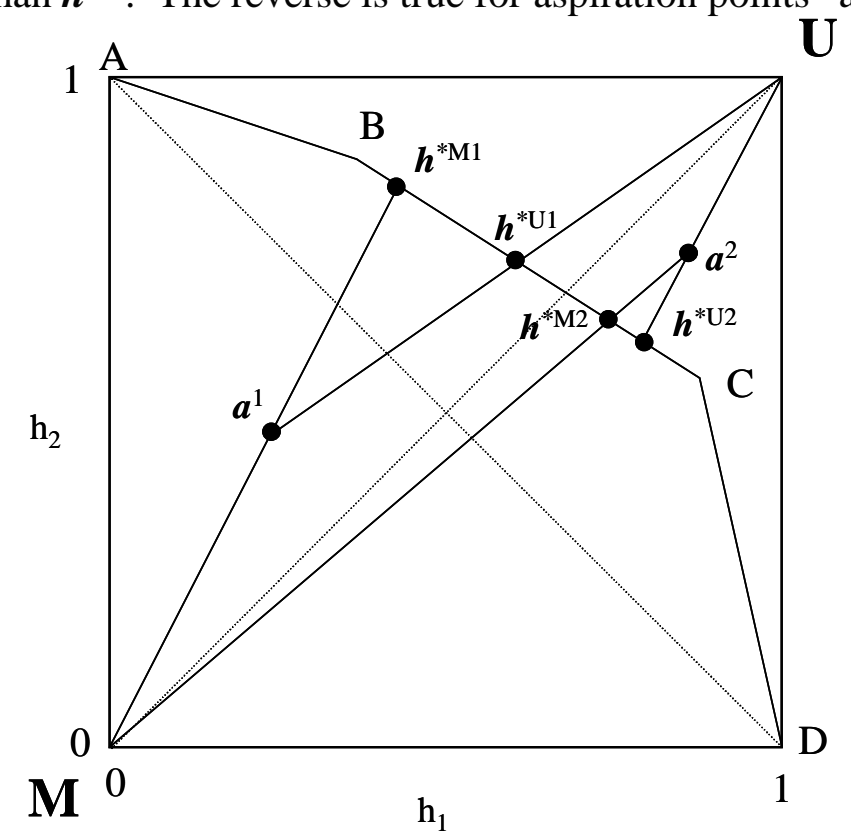

1. Comparing PM and PU in a two-dimensional polyhedral case

The PU formulation seeks a solution such that weighted deviations from the ideal point $\boldsymbol{U}$ are equal. In contrast, PM seeks a solution such that the weighted deviations from the nadir point $\boldsymbol{M}$ are equal. Both formulations are based on weighted deviations from a reference point. However, since the nadir point is the true zero point, the weighted deviations from this nadir point for PM are equivalent to weighted achievement. Figure 1 shows PM as "pushing out" from $\boldsymbol{M}$, and PU as "reaching down" from $\boldsymbol{U}$.

The relationship between aspiration points and weights is as follows. For a given aspiration point $\boldsymbol{a}=\left(a_{1}, a_{2}, \ldots, a_{q}\right)$, the weights $\boldsymbol{w}=\left(w_{1}, w_{2}, \ldots, w_{q}\right)$ are:

$\mathrm{PU}: w_{k}=\frac{1}{1-a_{k}}, k=1,2, \ldots, q: \quad \mathrm{PM}: w_{k}=\frac{1}{a_{k}}, k=1,2, \ldots, q$

These weights can be normalized so that they sum to unity although it is not necessary to do so. In this paper, however, our investigation is undertaken using aspiration points, not 
weights. The use of aspiration points better reflects the intent of most reference point methodologies. PU seeks to minimize the maximum weighted deviation, but so that the proportional deviations are equal; that is:

$$
\frac{1-h_{1}^{* U}}{1-a_{1}}=\frac{1-h_{2}^{* U}}{1-a_{2}}=\ldots=\frac{1-h_{q}^{* U}}{1-a_{q}},\left(1-a_{k}\right)>0, k=1,2, \ldots ., q
$$

PM seeks to maximize the minimum weighted achievement so that, if possible, proportional achievements are equal; that is:

$$
\frac{h_{1}^{* U}}{a_{1}}=\frac{h_{2}^{* U}}{a_{2}}=\ldots=\frac{h_{q}^{* U}}{a_{q}}, a_{k}>0, k=1,2, \ldots ., q
$$

There is, of course, no guarantee that these equalities will hold. Rather, these equations reflect the intent of the different formulations. In almost every case, for a given aspiration point, $\mathbf{a}=\left(a_{1}, a_{2}, \ldots, a_{\mathrm{q}}\right), \mathrm{PU}$ and PM result in different solutions.

\subsection{Aspiration Point Location}

We assume that all aspiration points have been scaled and are contained within the unit hypercube. The location of aspiration point $\boldsymbol{a}$ can be described relative to $N$, the set of nondominated solutions. First, $\boldsymbol{a}$ is either on $N$ or not. If not, $\boldsymbol{a}$ belongs to one of three mutually exclusive categories: under $N$, over $N$ or neither.

For either formulation (PM or PU), if solution $\boldsymbol{h}^{*}$ is a proportional solution, then the aspiration vector $\boldsymbol{a}$ will be either on, over or under $N$. When $\boldsymbol{h}^{* U}$ and $\boldsymbol{h}^{* \boldsymbol{M}}(\neq \boldsymbol{a})$ are both proportional and $\boldsymbol{a}$ is under $N, \boldsymbol{h}^{* U}$ will be closer to $\boldsymbol{L}$ than $\boldsymbol{h}^{* \boldsymbol{M}}$. The reverse holds when $\boldsymbol{a}$ is over $N$.

\section{Analysis of the Hyperspherical Case}

We now apply our analysis of discrepancy to the case where the set of nondominated solutions, $N$, is described by the boundary of the nonnegative section of a unit hypersphere centered at the origin. In two dimensions, this is the intersection of the boundary of a unit circle with the nonnegative quadrant, where the equation for $N$ is $h_{1}^{2}+h_{2}^{2}=1$.

This simplification of representing $N$ as the boundary of the nonnegative portion of a $q$-dimensional spheroid aids the ensuing analysis without any loss of generality, especially in higher dimensions. A hyperspherical approximation is one (symmetric) limit of a general polyhedral set, as the number of facets tends to infinity. Consequently, the analysis of differences between the two formulations is less affected by the "noise" of the nondominated surface. In addition to being smooth and symmetric, the hyperspherical approximation shares with the unit hypercube the $q$ extreme points which individually maximize each objective function over the unit hypersphere. Also, since the discrepancy associated with the hyperspherical approximation tends to be less than in the linear case, it is a conservative estimate of discrepancy. 


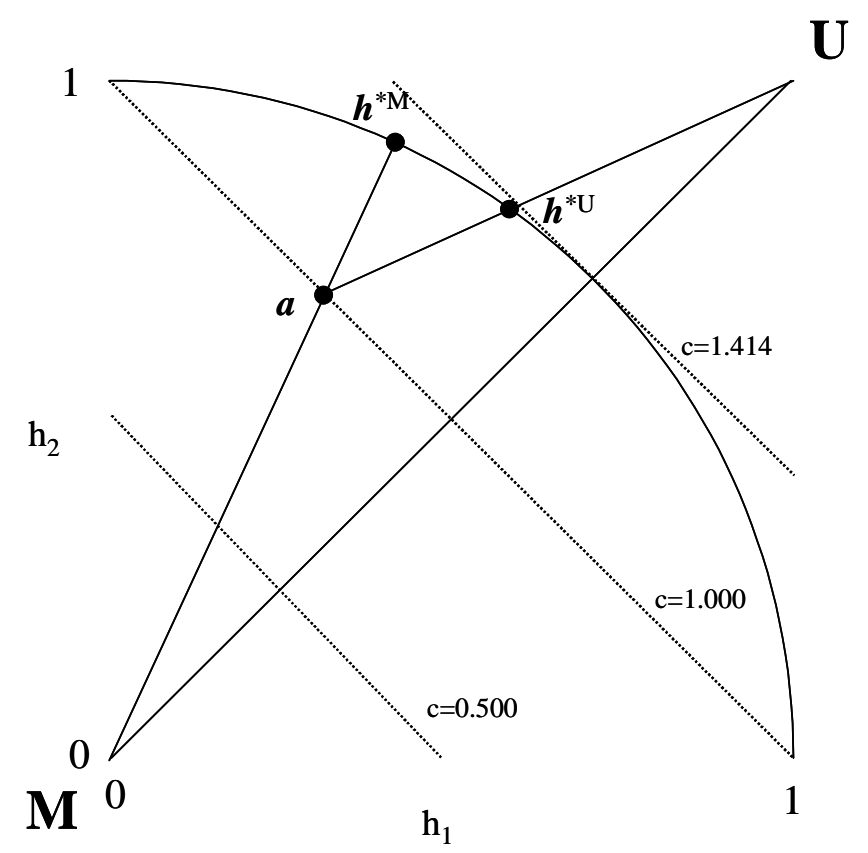

2. Two-dimensional hyperspherical case

We define $d_{h}$ as the Euclidean distance between the PM and PU solutions for any given $\boldsymbol{a}$ and use it as the measure of discrepancy. In order to explore how the amount of discrepancy depends on the location of $\boldsymbol{a}$, we examine how close $\boldsymbol{a}$ is to the line segment $\boldsymbol{L}=\boldsymbol{M U}$ and characterize the hyperplane containing $\boldsymbol{a}$. We define $d_{a L}$ as the Euclidean distance of $\boldsymbol{a}$ from $\boldsymbol{L}$ and let $c=h_{1}+h_{2}+\ldots+h_{q}$. Figure 2 illustrates these measures. Note that for $c \leq 1$, all corresponding aspiration points are on or under $N$. As $c$ approaches 0 , the aspiration points approach $\boldsymbol{M}$. For $c \geq 2 / \sqrt{2}$, all corresponding aspiration points are on or over $N$. As $c$ approaches 2, the aspiration points approach $\boldsymbol{U}$.

For $\boldsymbol{a}$ under $N$, as c increases and $\boldsymbol{a}$ is closer to the nondominated set, the discrepancy decreases. Choosing aspiration points very near to the ideal point $\boldsymbol{U}$ will result in considerable discrepancy, as also will aspiration points which are near $\boldsymbol{M}$.

Let us put the discrepancy measure in context. The maximum theoretically possible discrepancy in two dimensions, with a hyperspherical $N$, is 0.7654 . In this (unlikely) situation, for a given aspiration point, PU would give solution $(1,0)$ while PM would give solution $(1 / \sqrt{ } 2,1 / \sqrt{ } 2)$, or vice versa. Consider a "middle of the road" discrepancy of 0.2. What does this mean? For the case of $c=1$; that is, for all aspiration points on the hyperplane $h_{1}+h_{2}=1$, the median discrepancy is 0.21 , with a mean of 0.19 . A discrepancy of 0.2 is quite realistic. An example of a 0.2 discrepancy is shown in Table 3.

\begin{tabular}{cccccc}
\hline$a_{1}$ & $a_{2}$ & $h_{1}{ }^{* \mathrm{U}}$ & $h_{2}{ }^{{ }^{\mathrm{U}}}$ & $h_{1}{ }^{{ }_{\mathrm{M}} \mathrm{M}}$ & $h_{2}{ }^{{ }^{* \mathrm{M}}}$ \\
\hline 0.310 & 0.690 & 0.583 & 0.813 & 0.410 & 0.912 \\
\hline
\end{tabular}

3. Discrepancy Example where $\mathrm{d}_{\mathrm{h}}=0.2$ 
Thus, the solution is such that $h_{1}{ }^{* \mathrm{U}}$ is $42 \%$ larger than $h_{1}{ }^{* \mathrm{M}}$ while $h_{2}{ }^{{ }^{*} \mathrm{U}}$ is $89 \%$ of $h_{2}{ }^{* \mathrm{M}}$. Since the objective value has been normalized over the range [0,1], we have that $h_{1}{ }^{*}$ is $17.3 \%$ further along the range (i.e., $17.3 \%$ better achievement) than $h_{1}{ }^{*}$, while $h_{2}{ }^{* \mathrm{M}}$ is $9.9 \%$ further along the range than $h_{2}{ }^{*}$. Clearly, very different solutions result from the same aspiration point - depending entirely on the framing; that is, whether it is deviation from the ideal or achievement from the nadir.

\subsection{Dispersion of PU and PM solutions in three dimensions}

The behaviour of these two formulations can also be examined from the perspective of dispersion. That is, for a given set of aspiration points, what does the pattern of solutions produced by PU and by PM look like?

To examine this, we consider the three-dimensional case with equally spaced aspiration points within the unit cube. By projecting the unit cube onto the plane, a visual picture of the dispersion patterns for PU and PM can be obtained. Using a 0.05 grid, this represents $11 \times 11 \times 11=1,331$ aspiration points within the unit cube. Any of these aspiration points is therefore equally likely. Figure 4 shows the resulting dispersion of solutions over the spherical nondominated set for PM and PU. The perspective is that of looking straight down the ray $\boldsymbol{M U}$, from above the ideal point $\boldsymbol{U}$.
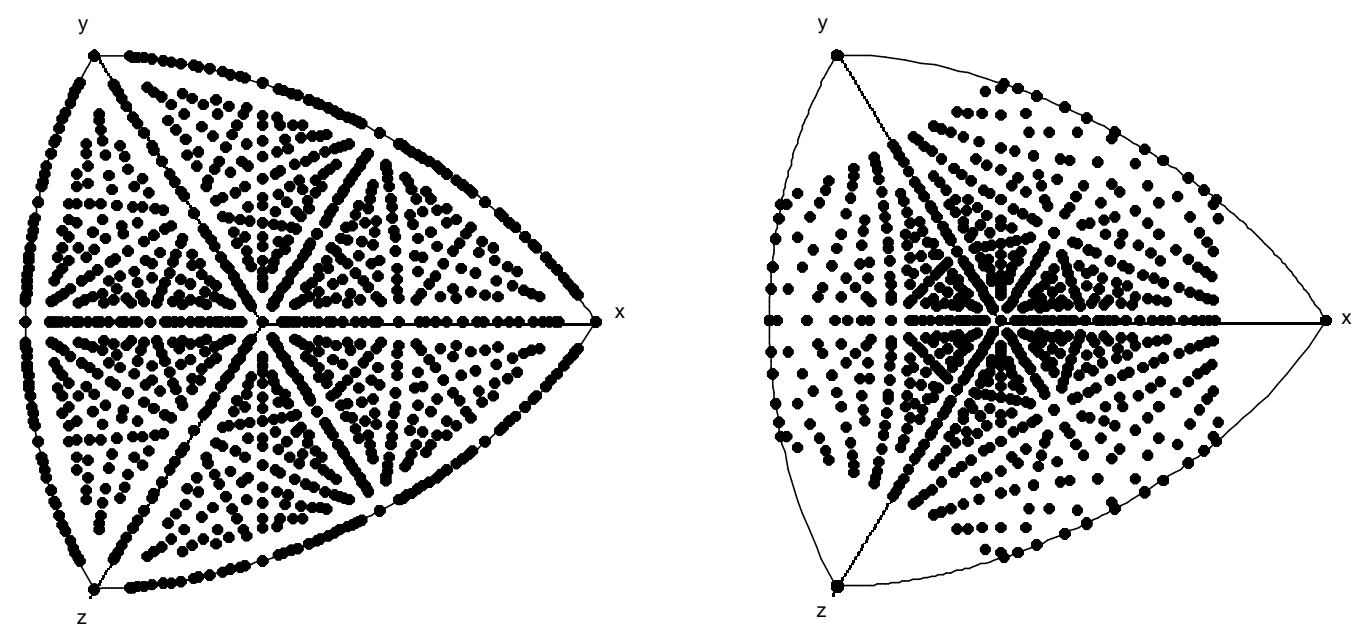

4. Two-dimensional projection of PM and PU dispersions respectively

The dispersion of, and by implication the discrepancy between, PU and PM solutions are different. Overall, PM provides a more even pattern of solutions. PU solutions are either toward the centre or to the absolute extremes. Although it cannot be seen from Figure 4 , the three extreme point solutions $(1,0,0),(0,1,0)$ and $(0,0,1)$ each comprise 100 solutions. For example, under PU there were 100 different aspiration points which all gave the same final solution of $(1,0,0)$. In three dimensions, $52 \%$ of the aspiration points are under $N$. 


\section{Experiment}

Differences between PM and PU are largely a function of the location of the aspiration point. Clearly, in any practical use of reference point methods, decision makers do not randomly choose aspiration points evenly throughout the unit hypercube. Perhaps in practice with actual aspiration points, these differences are not as significant? We therefore designed a simple experiment to examine this question and to also see which of the two formulations (PM or PU) decision makers preferred.

A production scheduling decision problem was used for the experiment. This simple problem has been used successfully in other experimental contexts by one of the authors (Corner and Buchanan, [3]). The nondominated set of the three objective production scheduling problem was chosen to be spherical.

A naive solution method, similar to the GUESS method [2], was used to solve the problem whereby the decision maker guesses a solution (the aspiration point) and the method finds a solution using both the PM and PU formulation. The decision maker chooses between the PM and PU solutions (or indicates indifference) and the method proceeds until a satisfactory solution is found. Although it was the goal of the participants to derive a good production schedule, we were only interested in the choice between the PM and PU solutions at each iteration. 58 students participated in the experiment which was treated as a case study and became part of their assessable work for a course; 38 were MBA students at Auburn University, while the remaining 20 were students from the University of Waikato.

The hypothesis was that participants would prefer PM to PU because, we assumed, decision makers are more "achievement-oriented" than "deviation-oriented." Because the production scheduling problem was formulated with a spherical nondominated set, PM will always produce a proportional solution. This is not the case for PU. We therefore eliminated all iterations where the PU solution was not proportional. The data was further reduced by eliminating all responses of indifference. The final data set contained aspiration points which resulted in a proportional PM or PU solution, between which participants expressed a clear preference.

\subsection{Results}

Aspiration points were distributed such that almost $75 \%$ were over $N$. This is not surprising - participants simply wanted greater achievement than was often feasible. If aspiration points were randomly distributed, then only $48.65 \%$ of aspiration points would be over $N$. The distribution of guesses, therefore, is not random $(\mathrm{p}=0.0000)$. With the raw data (before non-proportional PU solutions and indifference were excluded), $82 \%$ of aspiration points were over $N$.

Overall, $\mathrm{PM}$ is preferred at $57.0 \%(\mathrm{p}=0.0006)$. However, this overall preference for PM should be interpreted in the context of the aspiration point. If $\boldsymbol{a}$ is over $N$, PM is clearly preferred at $61.0 \%(\mathrm{p}=0.0000)$. If $\boldsymbol{a}$ is under $N, \mathrm{PU}$ is preferred at $55.2 \%$, although this is not statistically significant $(\mathrm{p}=0.2131)$. 


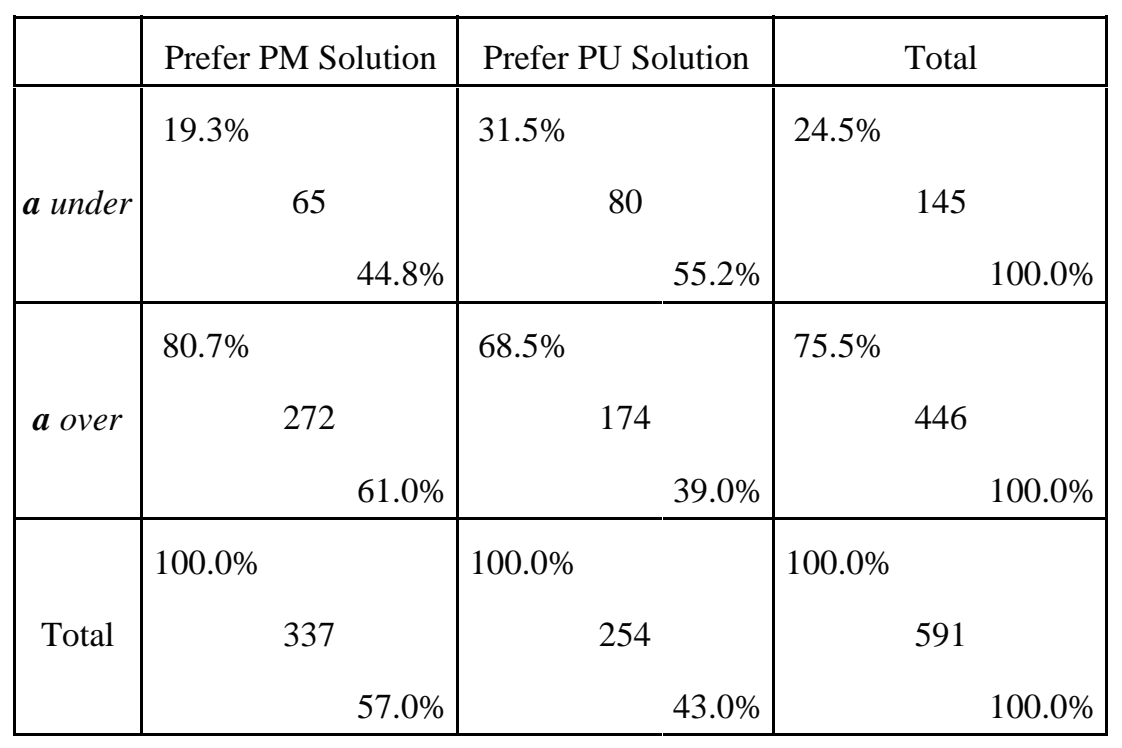

5. Categorization of aspiration points and solution choices

Recall that if the aspiration point is over $N$, then PM is closer to the center than PU; the converse is true for an aspiration point under $N$. The data above suggest that decision makers prefer solutions which are closer to the center, which represent more of a compromise. Certainly the achievement hypothesis, where decision makers are assumed to prefer an achievement-oriented approach, is rejected. Rather the position of the aspiration point (under $N$ or over $N$ ) and the apparent desire of decision makers for "centered" solutions would seem to explain the choice of solution. If this is accepted and given that most aspiration points are over $N$, then PM would be the solution method of choice. However, this should be qualified in two ways. First, if weights are used (i.e., aspiration points on the hyperplane $h_{1}+h_{2}+\ldots+h_{q}=1$ ), then because such aspiration points will always be under $N$, decision makers are likely to prefer PU. Second, if an analyst was seeking to encourage divergence rather than convergence, then PU is a better choice given that most aspiration points are over $N$.

\section{Conclusion}

The motivation for this paper came from an examination of reference point solution methods. Specifically, what is the effect of the choice of reference point? We limited our study to a simple Tchebycheff reference point formulation and considered both the ideal and nadir reference points. It has been shown that these two formulations (PU - reaching down and PM - pushing up) typically give different solutions for the same aspiration point. The choice of reference point influences the structure of the interactive solution model and may, perhaps, influence the behaviour of a decision maker.

The two formulations have different philosophies. PU seeks to minimize the maximum deviation; i.e., to make all weighted deviations equal, if possible. PM seeks to maximize the minimum achievement; i.e., to make all weighted achievements equal, if possible. We asked whether decision makers may be more comfortable with achievement, rather than 
deviation, oriented solution methods. In practice, the two formulations behave differently and their respective usefulness is contingent on what one is trying to achieve. If, for example, the aim is to generate a wider range of possible solutions then PU, with its typically greater dispersion, may be more appropriate.

The study shows that different solutions result from the same aspiration point, and that this difference can be significant. The magnitude of this difference depends on the location of the aspiration point and the shape of the nondominated surface. Particularly, if aspiration points are located near the nadir or ideal reference points and they are somewhat away from the center, then the two solutions are often considerably different.

A classification of aspiration points was developed as under $N$, over $N$ or neither. In general, if an aspiration point is under $N$ then the PU solution will be closer to the centre than the PM solution. Conversely, if the aspiration point is over $N$ then the PM solution will be closer to the centre than the PU solution. This result enables us to comment further. The congruence between the aspiration point and the resulting solution is also an important consideration, at least in an interactive reference point solution method. Thus, if the aspiration point is over $N$ and near to the ideal point, and PU is used, there will be considerable difference between the aspiration point and the resulting solution. This difference will also occur when PM is used, if the aspiration point is under $N$ and near the nadir point. Evidence from this experiment and other experiences with the reference point methods suggests that aspiration points will usually be close to the ideal point; decisions makers typically want more than they can get. This suggests that if some congruence is desired so that the resulting solution is somewhat similar to the aspiration point, then PM should be used.

The experiment showed that there is evidence of preference for one type of solution; however, this is not simply a preference for the results of one formulation over another. The preference appears, rather, to be related to the centrality of the resulting solution, suggesting that decision makers tend to seek compromise solutions, when given the opportunity. There appears to a tendency toward compromising among the objectives. Practically speaking, then, what should an analyst do? Recommend a method that will give a centralising tendency (assuming most aspiration points are over $N$ and close to the ideal point) and thereby support a decision maker in this direction? Or should the analyst recommend a method which counteracts this tendency of decision maker toward compromise in the hope that new and better solutions will be found?

Both methods have their advantages. Therefore we should make use of both methods; we should encourage decision makers, as Russo and Schoemaker [5] suggest, to use more than one frame of reference. The value comes not from choosing the "best" or most appropriate frame of reference, but from considering more than one perspective or frame. This paper has shown how these two different formulations (same scalarizing function but with different reference points) behave. They behave differently and both can be useful. It does not appear that the structure of the method significantly affects decision maker behaviour. It does not appear that decision makers are necessarily oriented toward either an achievement or a deviation focus. 


\section{References}

[1] Beach, L.R. and Mitchell, T.R. 1990, "Image theory: A behavioural theory of decisions in organisations," in Staw, B.M. and Cummings, L.L. (Eds), Research in Organisation Behaviour, Vol 12, JAI Press, Greenwich, CT.

[2] Buchanan, J. 1997, "A naive approach for solving MCDM problems: The GUESS method," Journal of the Operational Research Society, 48(2), 202-206.

[3] Corner, J.L. and Buchanan, J.T. 1997, "Capturing decision maker preference: Experimental comparison of decision analysis and MCDM techniques", European Journal of Operational Research, 98(1), 85-97.

[4] Korhonen, P. J. and Wallenius, J. 1988, “A Pareto race," Naval Research Logistics, $35(6), 615-623$.

[5] Russo, J.E. and Schoemaker, P.J.H. 1989, Decision Traps, Doubleday, New York.

[6] Simon, H.A. 1957, Models of Man, Macmillan, New York.

[7] Steuer, R. E. and Choo, E.-U. 1983, “An iteractive weighted tchebycheff procedure for multiple objective programming," Mathematical Programming, 26, 326-344.

[8] Tversky, A. and Kahneman, D. 1981. "The framing of decisions and the psychology of choice," Science, 211, 453-458.

[9] Wierzbicki, A. P. 1980, "The use of reference objectives in multiobjective optimization," Lecture Notes in Economics and Mathematical Systems, 177, 468-486. 